\title{
Neuraltherapie
}

\section{Lorenz Fischer}

Kollegiale Instanz für Komplementäre Medizin (KIKOM), Universität Bern, Inselspital, Imhoof-Pavillon, 3010 Bern, Schweiz,

fischer.lori@bluewin.ch

\section{Definition}

Die Neuraltherapie (hauptsächlich entwickelt von den Gebrüdern Huneke) ist eine Injektionsbehandlung, welche Lokalanästhetika zur Diagnostik und Therapie nutzt. Durch gezielte Ausschaltung bestimmter Strukturen ergibt sich eine präzise Diagnostik. Therapeutisch werden einerseits gezielt Reize gesetzt und andererseits neuronale Fehlleistungen unterbrochen (u.a. Circulus vitiosusim Schmerzgeschehen). Damit nutzt die Behandlungsmethode die regulatorischen Eigenschaften insbesondere des vegetativen Nervensystems hauptsächlich auf zwei Ebenen: zum einen über die Segmentreflektorik, zum anderen über das sog. Störfeld, das unabhängig von der segmentalen Zuordnung krankheitsauslösend oder -unterhaltend wirkt. Die Neuraltherapie (und Diagnostik) gliedert sich somit in:

- Lokale Therapie (z.B. Infiltrationen von Trigger-Punkten, Gelenken, Sehnenansätzen, peripheren Nerven) und Segment-Therapie (z.B. Quaddel-Therapie in den HEAD-Zonen, Umflutung vegetativer Ganglien, Nervenwurzeln).

- Störfeld-Therapie: Ein sog. Störfeld (Irritationszone) ist ein meist asymptomatischer, chronischer Reizzustand einer bestimmten Struktur (verschiedene Lokalisationen) des Organismus. Hiervon ausgehende Impulse können prinzipiell jedes andere System beeinflussen, unabhängig von der segmentalen Zuordnung. Es handelt sich oft um pathologische Veränderungen im Zahn-/Kieferbereich, chronische (oft asymptomatische) Entzündungen im Tonsillen- oder Nasennebenhöhlenbereich, bestimmte Narben usw.

\section{Material}

Ideal ist Procain 1\% (kurze Halbwertszeit und damit gut steuerbar, Abbau im Gewebe durch die Pseudocholinesterase, Durchblutungsförderung indirekt und direkt). Ebenfalls geeignet für die Neuraltherapie ist Lidocain $1 \%$.

\section{Indikationen}

Die Hauptindikationen sind akute und chronische Schmerzen am Bewegungsapparat. Sehr gute Indikationen sind auch Kopfschmerzen (siehe u.a. Lehrbücher sowie [1]), funktionelle Erkrankungen wie Colon irritabile oder chronisch entzündliche Erkrankungen (Autoimmunerkrankungen).

\section{Kontraindikationen}

Allergie gegen Lokalanästhetika (sehr selten bei Procain), Gerinnungsstörungen oder Antikoagulation, Tendenz zu vasovagalen Synkopen.

\section{Neurophysiologie und Wirkmechanismen}

Nozizeptive Vorgänge sind ohne Mitbeteiligung des Sympathikus nicht möglich. Sie lösen immer eine Reflexantwort aus. Diese kommt über Reflexbahnen zustande: kuti-viszeral, viszero-kutan, viszero-somatomotorisch usw. (Segmentreflektorik). Die vorwiegend sympathisch vermittelte Reflexantwort zeigt Durchblutungsveränderungen, Hautturgor-Erhöhungen, Hyperalgesie bestimmter Hautbezirke, Dysregulation des metamer zugehörigen inneren Organs sowie eine Erhöhung des Muskeltonus [2, 3]. Nozizeptive Afferenzen aus den Systemen Haut, Muskulatur und inneres Organ konvergieren auf dieselbe Hinterhornzelle des Rückenmarks. Die weitere Verschaltung erfolgt nun divergent: gleichzeitig über das Seitenhorn zum Sympathikus sowie über das Vorderhorn zur Skelettmuskulatur und zum Gehirn. So werden zum Beispiel sympathische und somatomotorische Kerne gleichzeitig erregt. Durch diese Verschaltungen kommt ein Circulus vitiosus (positive Rückkopplung, Iteration analog der Chaostheorie) im Schmerzgeschehen zustande [3]. Hauptvermittler ist der Sympathikus (Abb. 1). Der Circulus vitiosus wird verstärkt, indem der efferente (!) Sympathikus in der Peripherie unter pathologischen Bedingungen kurzschlussartig auf nozizeptive Afferenzen schalten kann: sympathisch-afferente Kopplung [4-7]. Sympathisch-afferente Kopplung und neuroplastische Veränderungen in Rückenmark und Gehirn bewirken eine zusätzliche, mehrfache Iteration im Schmerzgeschehen.

Zudem kann der Sympathikus unter pathologischen Bedingungen über eine Vasodilatation mit Plasmaextravasation und Sezernierung von proinflammatorischen Neuropeptiden aus seinen eigenen Nervenfasern eine neurogene Entzündung verursachen [8-10]. Diese Entzündung senkt die Reizschwelle der Nozizeptoren und rekrutiert gleichzeitig «schlafende» Nozizeptoren aus der Umgebung. Dadurch wird der Circulus vitiosus weiter verstärkt.

Tracey [11] beschreibt 2002 einen «inflammatorischen Reflex des vegetativen Nervensystems». Dieser reguliert

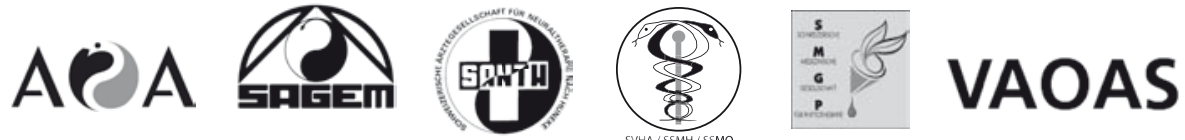


reflektorisch Entzündungs- und Immunantworten des Organismus.

Ricker [12] konnte bereits 1924 tierexperimentell zeigen, dass auch eine weit zurückliegende pathologische Reizung des Sympathikus engrammatisch gespeichert wird. Der Sympathikus hat demnach eine Art "Gedächtnis» für pathologische Reize.

In diese verschiedenen Ebenen der Neurophysiologie des Schmerzes kann mit der Neuraltherapie direkt und logisch eingegriffen werden [3,13]: Neuraltherapie bedeutet einerseits Impulssetzung (Nadelstich), andererseits Unterbrechung (Lokalanästhetikum) einer pathologischen Belastung. Durch wiederholte Anwendung kann die «Löschung» der engrammatischen pathologischen Reizbarkeit des Sympathikus und die Normalisierung der Gewebeperfusion erreicht werden. Die kurze Unterbrechung der ineinander greifenden sympathischen und somatischen Reflexbogen, welche sich beim Schmerz- und Entzündungsgeschehen in einen Circulus vitiosus mittels positiver Rückkopplung steigern, kann dazu führen, dass die Systeme autoregulatorisch wieder die physiologische «Mitte» erreichen. Zudem kann die sympathisch-afferente Kopplung prinzipiell aufgehoben werden. Der Circulus vitiosus «Nozizeptorenaktivität - Sympathikuserregung - Zirkulationsstörung - Neurogene Entzündung - Muskelverspannungen usw.» kann an verschiedenen Stellen gleichzeitig mit dem Lokalanästhetikum unterbrochen werden: z.B. mit Infiltrationen in den myofaszialen Trigger-Punkt, in Hautquaddeln, Injektionen an vegetative Ganglien, Injektionen an den perivasalen Sympathikus usw.

Zudem konnte Cassuto [8] 2006 zeigen, dass wiederholte Lokalanästhetika die neurogene Entzündung lindern können. Zusätzlich kann durch Nadelstich und Lokalanästhetikum ein günstiger Effekt auf die Hinterhorn-Eingangskontrolle des Rückenmarks [14] erreicht werden.

\section{Anwender}

Die lokale und segmentale Neuraltherapie ist sowohl im diagnostischen als auch im therapeutischen Bereich integraler Bestandteil der täglichen Arbeit jeder Schmerzklinik, von Rheumatologen, von Orthopäden und auch von Allgemeinmedizinern und wird in der Schweiz und in den angelsächsischen Ländern meist als «diagnostische» und «therapeutische Lokalanästhesie» bezeichnet. Dieser Teil der Neuraltherapie gilt als Schulmedizin und wird über die Grundversicherung abgerechnet.

Der Zusatz «nach Huneke» wird dann verwendet, wenn Störfelder in die Therapie miteinbezogen werden. Nur dieser spezielle Teil der Neuraltherapie gilt als Komplementärmedizin und wird über die Zusatzversicherung abgerechnet.

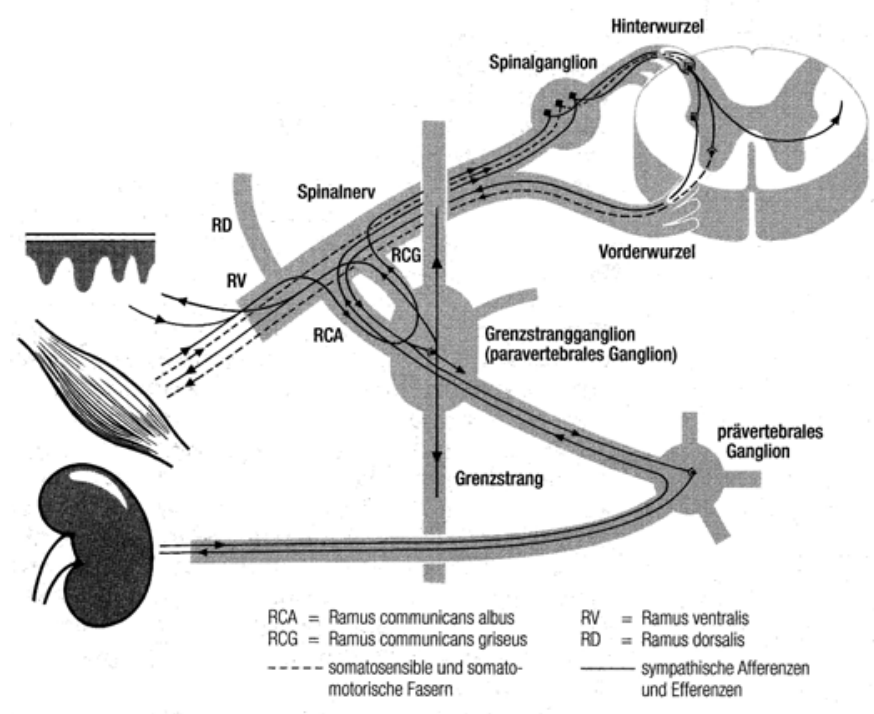

Abb. 1. Reflektorische Verschaltung von Haut, Muskulatur und innerem Organ. Schematische Darstellung. Kombinationsmöglichkeiten von Injektionen mit Lokalanästhetika sind hier sichtbar: Hautquaddeln, Injektion in die Muskulatur (Trigger-Punkte), Injektionen an den Spinalnerv, an das para- und prävertebrale Ganglion. Durch die Unterbrechung eines Circulus vitiosus kann eine lang anhaltende Wirkung erzielt werden. Quelle: Fischer L: Neuraltherapie nach Huneke. Neurophysiologie, Injektionstechnik und Therapievorschläge, ed 3. Stuttgart, Hippokrates, 2007.

Für die Neuraltherapie nach Huneke existiert ein Fähigkeitsausweis FMH/SANTH (Schweizerische Ärztegesellschaft für Neuraltherapie nach Huneke); zurzeit sind in der Schweiz 116 Ärztinnen und Ärzte zertifiziert.

\section{Forschung}

Die Forschung befasst sich einerseits mit Wirkmechanismen (neueste Arbeit: Pfister und Fischer, 2009 [13]), andererseits mit klinischen Studien:

Im Rahmen der Evaluation der Verfahren durch das BAG (Bundesamt für Gesundheit) entstand die folgende Studie über die Wirksamkeit:

- Mermod J, Fischer L, Staub L, Busato A. BMC Complement Altern Med 2008;8:1-10. www.biomedcentral. com/1472-6882/8/33.

Diese grosse Studie mit insgesamt 405 Patienten wurde 2008 einerseits als Dissertation von der Universität Bern anerkannt, andererseits «peer-reviewed» publiziert. Sie zeigt signifikant bessere Resultate bei Patienten, die mit Neuraltherapie behandelt wurden (gegenüber konventioneller Behandlung). In der Neuraltherapie-Gruppe mussten auch weniger Arbeitsunfähigkeiten verschrieben werden. 
Ebenfalls in diesem Rahmen entstand eine Kostenstudie, ebenfalls als Dissertation anerkannt:

- Bissig P, Schoeni-Affolter F, Fischer L, Busato A: Is Neural Therapy Cheaper than Conventional Medicine? A Comparison of Cost Structure in Swiss Primary Care Providers - an Observational Study. Dissertation, Universität Bern, 2008.

Verglichen wurden rein konventionell-medizinische Praxen mit solchen, welche die Neuraltherapie integriert haben. Es wurden die Daten von insgesamt $4103 \mathrm{~Pa}$ tienten ausgewertet. Bei den jährlichen Gesamtkosten fand sich keine Differenz, jedoch in der Kostenstruktur: Beispielsweise benötigten Neuraltherapie-Patienten signifikant weniger Medikamente.

Eine weitere Studie zur Wirksamkeit der Neuraltherapie wurde 2007 in der Schweiz publiziert:

- Fischer L, Pfister M. Schweiz Zschr Ganzheitsmedizin 2007;19:30-35.

Eingeschlossen waren von Ärzten schriftlich zugewiesene Schmerzpatienten zur Neuraltherapie, welche vor der Neuraltherapie sechs Jahre an der Schmerzkrankheit litten und therapieresistent auf konventionellmedizinische Massnahmen waren. Zwei Drittel der Patienten erfuhren eine lang anhaltende Schmerzreduktion mit mehrmonatiger Beobachtungszeit; nur ein Fünftel blieb therapieresistent. Im Durchschnitt waren insgesamt lediglich 8,2 neuraltherapeutische Konsultationen notwendig. Bei mehr als der Hälfte der Patienten blieb der Schmerzmittelverbrauch auch nach mehrmonatiger Beobachtungszeit niedriger.
Weitere Studien und publizierte Kasuistiken sind zu finden im sog. Health Technology Assessment (HTA) über Neuraltherapie zuhanden des Bundesamtes für Gesundheit (Fischer L, Barop H, Maxion-Bergemann S; 2005).

\section{Zusammenfassung}

Die Interventionen der Neuraltherapie können als praktische Umsetzung der experimentellen und theoretischen Erkenntnisse der modernen Schmerzforschung angesehen werden, wenngleich die Methode vor Jahrzehnten empirisch entstanden ist. In diesem Sinne stellt die Neuraltherapie in vielen Fällen eine aufgrund der Pathomechanismen kausale Therapie dar.

\section{Ausbildungsmöglichkeiten}

Erstmals gehört die Neuraltherapie an der Universität Bern zu den Pflichtfächern für alle Studierenden (mit Prüfungsfragen).

Der Fähigkeitsausweis Neuraltherapie SANTH/FMH ist gebunden an einen FMH-Titel bzw. eine fünfjährige Weiterbildung an den von der FMH anerkannten Weiterbildungsstätten. Darauf bauen 157 Stunden des Fähigkeitsprogramms (Kurse) auf. Zudem besteht eine obligatorische Fortbildung: 30 Stunden pro 3 Jahre.

\section{Kontakt}

Schweizerische Ärztegesellschaft für Neuraltherapie nach Huneke SANTH Postfach 590

CH-3000 Bern 7

info@neuraltherapieschweiz.ch

\section{Lehrbücher}

Barop H: Lehrbuch und Atlas der Neuraltherapie nach Huneke. Stuttgart, Hippokrates, 1996.

Fischer L: Neuraltherapie nach Huneke. Neurophysiologie, Injektionstechnik und Therapievorschläge, ed 3. Stuttgart, Hippokrates, 2007.

\section{Literatur}

1 Maizels M, Scott B, Cohen W, Chen W: Intranasal lidocaine for treatment of migraine: a randomized, double-blind, controlled trial. JAMA 1996;276:319-321.

2 Fischer L: Myofasciale Trigger-Punkte und Neuraltherapie nach Huneke. Erfahrungsheilkunde 1998;3:117-126.

3 Fischer L: Pathophysiologie des Schmerzes und Neuraltherapie. Praxis 2003;92:2051-2059.

4 Baron R, Jänig W: Schmerzsyndrome mit kausaler Beteiligung des Sympathikus. Anästhesist 1998;47:4-23.

5 Baron R, Jänig W: Sympathetically maintained pain; in Bountra C, Muglani R, Schmidt W (eds): Pain. New York, Marcel Dekker, 2003, pp 309-320.
6 Jänig W, Baron R: Complex regional pain syndrome: mystery explained? Lancet Neurol 2003;2:687-697.

7 Jänig W: The Integrative Action of the Autonomic Nervous System. New York, Cambridge University Press, 2006.

8 Cassuto D, Sinclair R, Bonderovic M: Anti-inflammatory properties of local anesthetics and their present and potential clinical implications. Acta Anesthesiol Scand 2006;50:265-282.

9 Goadsby P, Edvinson L: The trigeminovascular system and migraine: studies characterizing cerebrovascular and neuropeptide changes seen in humans and cats. Ann Neurol 1993;33:48-56.

10 Strittmatter M, Grauer MT, Fischer C, Hammann G, Hoffmann KH, Blaes F, Schimrigk K:
Anatomic nervous system and neuroendocrine changes in patients with idiopathic trigeminal neuralgia. Cephalalgia 1996;16:476-480.

11 Tracey KJ: The inflammatory reflex. Nature 2002;420:853-859.

12 Ricker G: Pathologie als Naturwissenschaft Relationspathologie. Berlin, Springer, 1924.

13 Pfister M, Fischer L: Die Behandlung des komplexen regionalen Schmerzsyndroms der oberen Extremität mit wiederholter Lokalanästhesie des Ganglion stellatum. Praxis 2009;98:247-257.

14 Melzack R, Wall PD: Pain mechanisms: a new theory. Science 1965;150:971-979.

\section{Agenda UNION}

\section{Vorstandssitzung UNION \\ Delegiertenversammlung UNION \\ Vorstandssitzung UNION}

Donnerstag, 15. April 2010 (Vormittag); Vatter Business Center, Bern

Donnerstag, 15. April 2010 (Nachmittag); Vatter Business Center, Bern

Donnerstag, 24. Juni 2010 (Nachmittag); Hotel Kreuz, Bern 